\title{
Potência muscular máxima na flexão do cotovelo uni e bilateral
}

\author{
Roberto Simão ${ }^{1}$, Walace David Monteiro ${ }^{1,2}$ e Claudio Gil Soares Araújo ${ }^{1,3}$
}

\section{RESUMO}

Fundamentação: Exercícios de fortalecimento muscular podem ser realizados de forma uni e bilateral, contudo, pouco é sabido sobre a potência muscular (PM) e a carga máxima (CM) nessas condições. Objetivo: Comparar a PM e a CM na flexão do cotovelo entre os dois braços e entre a soma (das ações unilaterais e os resultados obtidos pela execução simultânea do gesto motor). Desses dois resultados com aquele obtido simultaneamente pelos dois braços. Metodologia: Submetemos 24 adultos jovens (14 homens) (PAR-Q negativo) e inexperientes no exercício de flexão de cotovelo ao teste de $1 \mathrm{RM}$ - duas repetições em velocidade máxima na fase concêntrica com $3 \mathrm{~s}$ de intervalo para cada carga -, com medida da potência (Fitrodyne, Bratislava), em cada braço e nos dois simultaneamente, em ordem randômica, para determinar a PM e a CM. Metodologia: 24 adultos jovens (14 homens) (PAR-Q negativo) foram submetidos ao teste de 1-RM (exercício flexão de cotovelo) com o objetivo de avaliarmos a PM e CM, todos sem experiência prévia no exercício proposto. O protocolo consistia de duas tentativas, com intervalo de $3 \mathrm{~s}$ entre as mesmas, quando o avaliado procurava imprimir o máximo de velocidade possível na fase concêntrica do movimento, utilizando ações unilaterais e bilaterais definidas randomicamente. Utilizou-se para medir a potência em cada braço e nos dois simultaneamente o Fitrodyne (Bratislava). Resultados: Os resultados para braço esquerdo e direito na CM $29,3 \pm 2,8$ e $29,7 \pm 2,9 \mathrm{~kg}-$ e na $\mathrm{PM}-106 \pm 14$ e $109 \pm$ $12 \mathrm{~W}$ - foram similares $(\mathrm{p}>0,05)$ e fortemente associados $(p>0,94)$. Comparando a soma dos valores unilaterais com

\footnotetext{
1. Programa de Pós-Graduação em Educação Física/Universidade Gama Filho.

2. Laboratório de Fisiologia do Exercício/Nuicaf - Aeronáutica.

3. Clinimex - Clínica de Medicina do Exercício.

Recebido em: 2/8/2001.
}

Aceito em: 24/9/2001.

Endereço para correspondência:

Prof. Dr. Claudio Gil S. Araújo

Rua Siqueira Campos, 93/101

22031-070 - Rio de Janeiro, RJ, Brasil

E-mail:cgaraujo@iis.com.br os da execução bilateral, a CM era $5 \%$ maior $(p=0,02)$ e a PM 5\% menor $(p=0,053)$. Conclusão: Apesar de todos serem destros, não houve diferenças unilaterais em CM e PM, provavelmente devido à inexperiência nesse exercício (fatores neurais). A soma dos resultados unilaterais difere em 5\% daquele obtido bilateralmente, mostrando, contudo, tendências opostas entre PM e CM, provavelmente refletindo uma limitação central na coordenação motora de um movimento complexo feito em máxima velocidade e com carga relativamente alta.

Palavras-chave: Potência muscular máxima. Exercícios unilaterais e bilaterais. Treinamento contra-resistência. Fortalecimento muscular. Déficit bilateral.

\section{ABSTRACT}

\section{Maximal muscle power in unilateral and bilateral elbow flexion}

Rationale: muscular strength exercises may be performed both unilaterally and bilaterally. However, little is known about muscular power (MP) and maximum load (ML) in these situations. Purpose: To compare MP and ML in elbow flexion between the two arms separately, and between the sum of these two results and the result of the exercise performed with both arms simultaneously. Methodology: Twenty-four young adults (14 male and 10 female; negative $P A R-Q$ ) with no experience in the elbow flexion test were submitted to the 1 RM test - two repetitions in maximum speed at the concentric phase, with $3 \mathrm{~s}$ interval for each load, measuring the power (Fitrodyne, Bratislava) in each arm and in both arms simultaneously on a randomized basis, in order to determine MP and ML. Results: The results for MP in left and right arm $-29.3 \pm 2.8$ and $29.7 \pm$ $2.9 \mathrm{~kg}$, respectively, and for $M L, 106 \pm 14$ and $109 \pm 12 \mathrm{~W}$, respectively - were similar ( $p>0.05)$ and strongly associated $(r=0.94)$. Comparing the sum of the unilateral values to the values of the bilateral exercise, ML was $5 \%$ high$\operatorname{er}(p=0,02)$, and MP was 5\% smaller $(p=0,053)$. Conclusion: While all subjects were right-handed, there was no unilateral difference in $M L$ and MP, probably due to their lack of experience in this exercise. The sum of unilateral results differs in about $5 \%$ of the bilateral results. There is, 
however, opposite trends between MP and ML, probably a reflex of central limitation in the motor coordination of a complex movement, performed with maximum speed and with a relatively high load.

Key words: Maximum muscular power. Unilateral and bilateral exercises. Counter-resistance training. Muscular strengthening. Bilateral deficit.

\section{INTRODUÇÃO}

Muitos são os trabalhos que evidenciam a importância da força e potência muscular ${ }^{1-4}$. Os treinamentos dessas variáveis têm demonstrado serem efetivos na melhoria de várias capacidades funcionais, bem como no aumento da massa muscular ${ }^{1,5}$. Tal fato os torna imprescindíveis na prescrição do treinamento direcionado a distintas populações, como, por exemplo, atletas, indivíduos que apresentam lesões ortopédicas, idosos, ou mesmo aqueles praticantes saudáveis que visam a promoção da saúde ${ }^{3,5}$.

Para trabalhar as variáveis citadas, a condução do treinamento pode ser realizada de forma bilateral ou unilateral. A força desenvolvida durante ações bilaterais é menor do que a soma da força desenvolvida unilateralmente ${ }^{6,7}$. Segundo alguns autores, essa diferença, chamada de déficit bilateral ${ }^{8}$, está associada com a estimulação reduzida de unidades motoras, que poderia ser causada pela inibição dos mecanismos protetores, resultando em uma menor produção de força ${ }^{9-11}$. É importante ressaltar que o treinamento com ações musculares simultâneas reduz o déficit bilateral, em relação à soma unilateral da força ${ }^{8,12,13}$. Embora atividades bilaterais reduzam o déficit, o desempenho em exercícios unilaterais pode constituir-se em uma estratégia importante, no sentido de conservar a força ${ }^{12,13}$, especialmente em situações de assimetria importante.

Dessa forma, este estudo objetiva: a) comparar a potência muscular máxima e a carga máxima, obtidas através de trabalho unilateral; b) comparar a soma da potência muscular máxima e da carga máxima obtida com cada um dos membros separadamente com aquela obtida no trabalho bilateral simultâneo.

\section{MÉTODOS}

Participaram deste estudo 24 indivíduos (10 mulheres) com idades entre 18 e 37 anos (26,5 $\pm 5,8$ anos), peso corporal entre 50 e $91 \mathrm{~kg}(71 \pm 13 \mathrm{~kg})$ e estatura entre $157 \mathrm{e}$ $187 \mathrm{~cm}(171,4 \pm 8,1 \mathrm{~cm})$. Dos 24 avaliados, 21 (oito mulheres) nunca haviam praticado exercícios com pesos, e três (duas mulheres) não participavam desse tipo de treinamento há pelo menos um ano.

Todos os indivíduos avaliados eram fisicamente ativos, já que se exercitavam pelo menos três vezes semanais ${ }^{14}$.
Antes da coleta de dados, todos responderam negativamente aos itens do questionário PAR- $\mathrm{Q}^{15} \mathrm{e}$ assinaram um termo de consentimento. A coleta constou das seguintes etapas: a) medidas do peso corporal e estatura; b) aplicação do teste de uma repetição máxima $(1 \mathrm{RM})^{16} \mathrm{em}$ velocidade máxima na fase concêntrica, objetivando determinar a carga que gerava a maior potência muscular e a carga máxima na flexão de cotovelo uni e bilateral.

Com objetivo de reduzir a margem de erro no teste de $1 \mathrm{RM}$, adotaram-se as seguintes estratégias ${ }^{17}$ :

1) Instruções padronizadas foram oferecidas antes do teste, de modo que o avaliado estivesse ciente de toda a rotina que envolvia a coleta de dados.

2) O avaliado foi instruído sobre a técnica de execução do exercício, inclusive realizando-o algumas vezes sem carga, para reduzir um possível efeito do aprendizado nos escores obtidos.

3) $\mathrm{O}$ avaliador estava atento quanto à posição adotada pelo praticante no momento da medida. Pequenas variações no posicionamento das articulações envolvidas no movimento poderiam acionar outros músculos, levando a interpretações errôneas dos escores obtidos.

4) Os pesos e a barra de ferro utilizados no estudo foram previamente aferidos contra balança calibrada.

Para estabelecer a carga que gerava a maior potência muscular e a carga máxima no teste de 1RM, utilizou-se o equipamento cross-over da marca Life Fitness modelo 2000, sendo executado em sua roldana baixa. Os implementos de carga obedeceram à sobrecarga do próprio aparelho (em forma de placas), cada uma delas com peso de cinco quilogramas. Além disso, quando necessário, foram implementadas cargas adicionais com anilhas de dois quilos, marca Ivanko. O exercício foi realizado com uma barra de ferro de apoio unilateral e bilateral, ambas da marca Buick. Para obtenção das medidas de potência muscular máxima e carga máxima, no exercício selecionado, fixou-se um tensiômetro Fitrodyne (Bratislava, Eslováquia) na placa localizada ao centro do aparelho. Dessa forma, em cada execução na fase concêntrica, o equipamento registrava a velocidade média alcançada, calculando a potência média em watts a partir do peso previamente digitado no mostrador do equipamento.

Para melhor discriminar a realização do exercício, estabeleceram-se as seguintes etapas de execução: posição inicial, fase concêntrica e fase excêntrica. A descrição detalhada nos exercícios em cada fase é apresentada a seguir:

1) Flexão do cotovelo em pé (bilateral).

A) Posição inicial - O indivíduo em pé, pernas paralelas com um pequeno afastamento lateral, com os joelhos estendidos, quadris na posição anatômica, braços ao longo do corpo com as mãos supinadas segurando a barra e a 

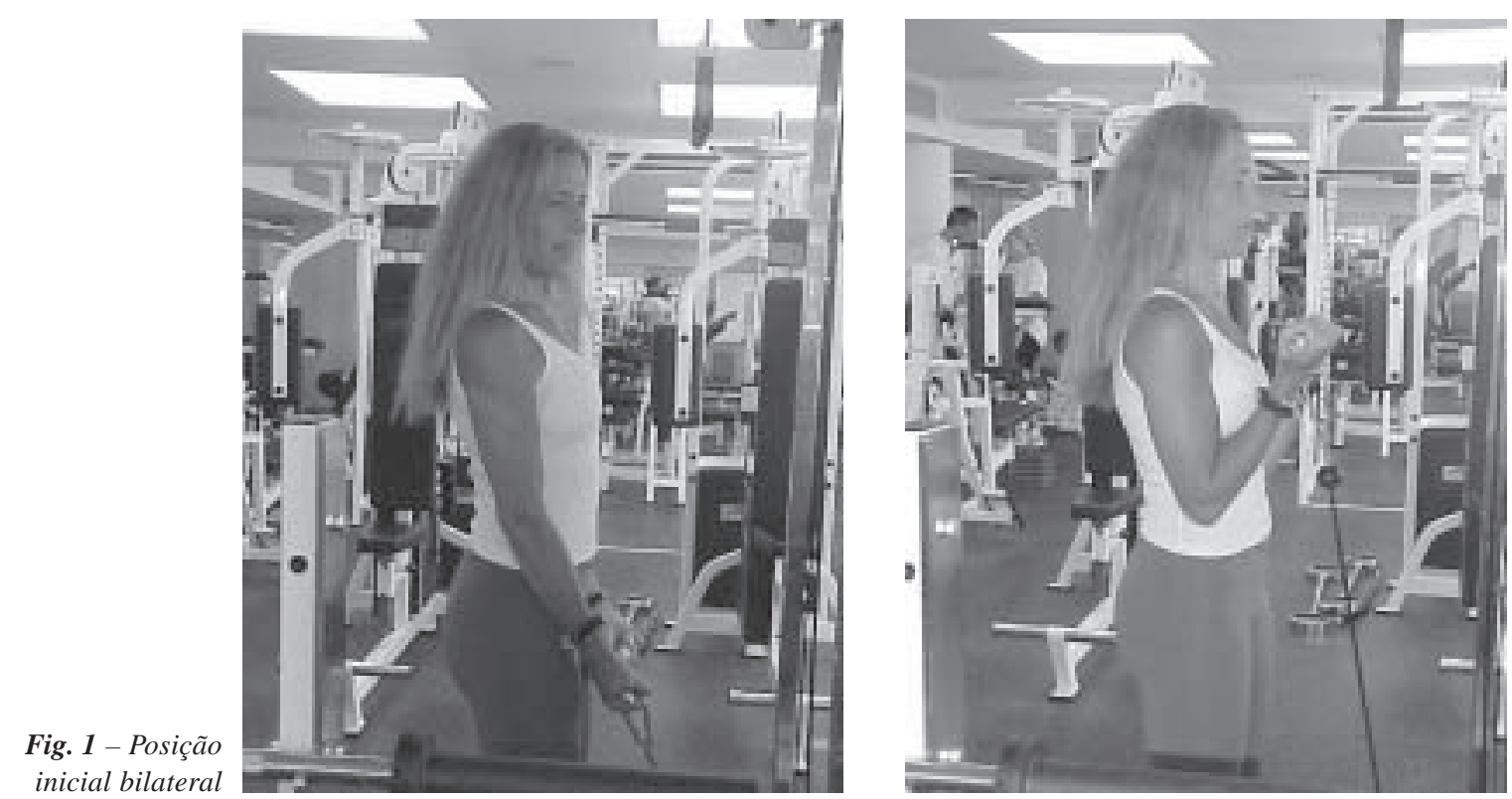

Fig. 2 - Fase concêntrica inicial bilateral
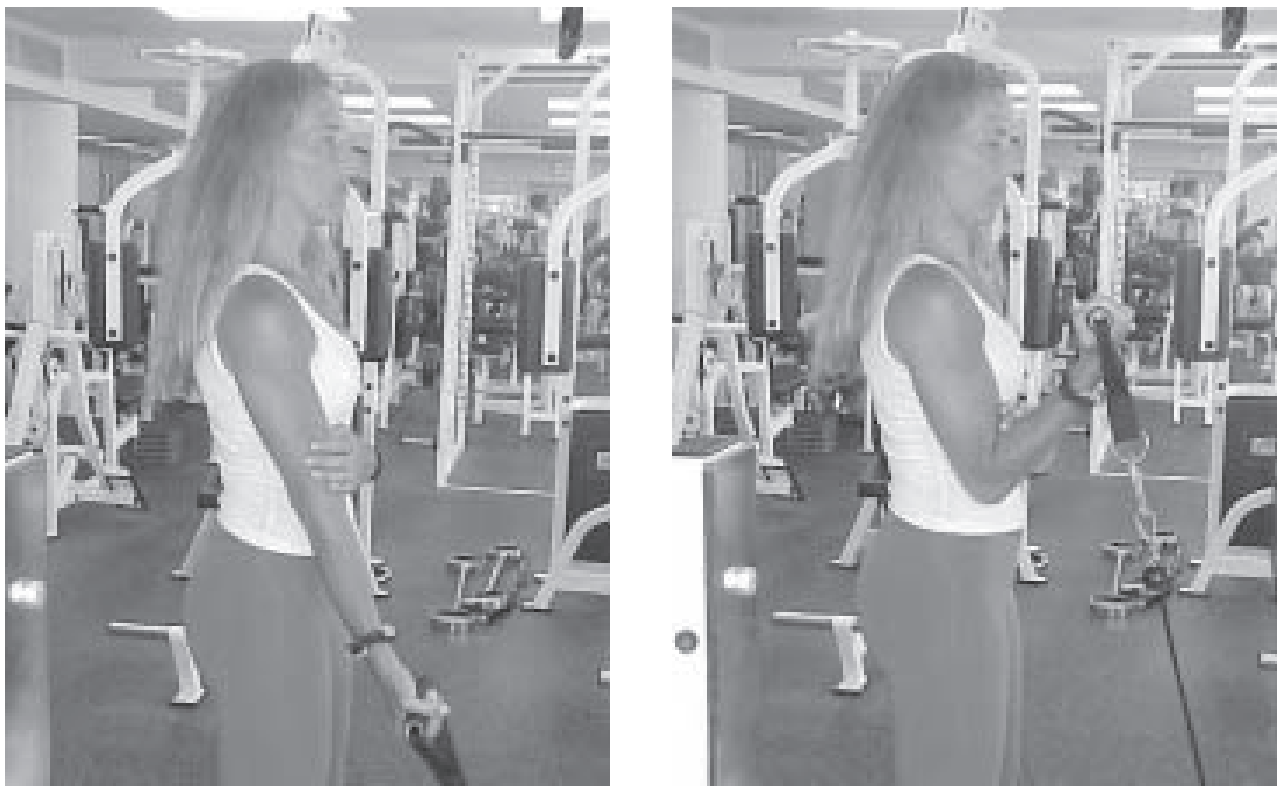

Fig. 4 - Fase concêntrica unilateral

cabeça posicionada com o plano de Frankfurt ${ }^{18}$. O posicionamento das mãos na barra para cada avaliado foi padronizado a partir do afastamento das mesmas, como demonstrado na figura 1.

B) Fase concêntrica - A partir da posição inicial, realizava-se a flexão completa dos cotovelos, como demonstrado na figura 2.

C) Fase excêntrica - A partir do final da fase concêntrica, os cotovelos voltam à posição inicial.

2) Flexão do cotovelo em pé (unilateral).

A) Posição inicial - O indivíduo em pé, pernas paralelas com um pequeno afastamento lateral, com os joelhos es-

tendidos, quadris na posição anatômica, braço ao longo do corpo com a mão supinada segurando a barra e a cabeça posicionada com o plano de Frankfurt ${ }^{18}$. O posicionamento da mão na barra para cada avaliado, foi padronizado ao lado do corpo (figura 3 ).

B) Fase concêntrica - A partir da posição inicial, realizava-se a flexão completa do cotovelo (figura 4).

C) Fase excêntrica - A partir do final da fase concêntrica, o cotovelo volta à posição inicial.

Para condução dos testes, os indivíduos foram divididos em dois grupos, selecionados aleatoriamente para o teste de 1 RM. O primeiro grupo realizava os exercícios na se- 
qüência: braço direito, braço esquerdo e ambos os braços. Já o segundo, realizava na seqüência: ambos os braços, braço esquerdo e braço direito. Ao iniciar o teste, cada avaliado realizava duas repetições, com três segundos de intervalo entre as repetições, imprimindo velocidade máxima na fase concêntrica do exercício. A maior potência muscular obtida nas duas repetições mensuradas pelo $\mathrm{Fi}$ trodyne, era considerada como sendo de melhor performance. A cada nova série, realizavam-se incrementos progressivos de $2 \mathrm{~kg}$, sendo dado um intervalo de um minuto entre cada série. Após a obtenção da carga máxima no teste de $1 \mathrm{RM}$, era dado um intervalo de descanso de cinco a dez minutos entre os testes.

O procedimento do teste de 1RM tinha o propósito de obter duas cargas: a que gerava a maior potência muscular e a carga máxima. Quando o avaliado não conseguia mais realizar o movimento completo de forma correta, o teste era interrompido. Desse modo, validou-se como carga máxima a que foi obtida na última execução correta.

A análise estatística foi realizada pelo teste- $t$ emparelhado, objetivando verificar a existência de diferença significativa entre os membros, bem como no somatório dos dois membros separadamente em relação ao trabalho realizado bilateralmente. Para todos os procedimentos considerou-se 5\% de significância.

\section{RESULTADOS}

A tabela 1 fornece a estatística descritiva na comparação da potência muscular do braço esquerdo e direito. Não há diferença significativa entre as duas medidas $(\mathrm{p}=0,35)$

\begin{tabular}{|c|c|c|c|c|c|c|c|}
\hline \multicolumn{8}{|c|}{$\begin{array}{c}\text { TABELA } 1 \\
\text { Comparação da potência muscular } \\
\text { máxima entre os membros }\end{array}$} \\
\hline Grupo & $\mathbf{N}$ & Média & DP & Mediana & Mínimo & Máximo & EPM \\
\hline Esquerdo & 24 & 106,7 & 70,7 & 91,5 & 27 & 268 & 14,4 \\
\hline Direito & 24 & 108,6 & 62,4 & 99 & 30 & 256 & 12,7 \\
\hline \multicolumn{8}{|c|}{ DP - desvio-padrão; EPM - erro padrão da média } \\
\hline \multicolumn{8}{|c|}{$\begin{array}{c}\text { TABELA } 2 \\
\text { Comparação do somatório unilateral com } \\
\text { o bilateral na potência muscular máxima }\end{array}$} \\
\hline Grupo & $\mathbf{N}$ & Média & DP & Mediana & Mínimo & Máximo & EPM \\
\hline Unilateral & 24 & 211,1 & 133,6 & 173,5 & 57 & 524 & 27,2 \\
\hline Bilateral & 24 & 230,3 & 136,3 & 212 & 71 & 604 & 27,8 \\
\hline
\end{tabular}

e a correlação entre elas é muito alta $(r=0,94)$. A tabela 2 descreve a comparação do somatório da potência muscular dos membros, com a potência muscular desenvolvida bilateralmente, em que as diferenças tendem à significância $(\mathrm{p}=0,053)$ e a associação entre as medidas é muito alta $(\mathrm{r}=0,91)$.

Embora em 62,5\% dos indivíduos o somatório da potência muscular na execução unilateral tenha sido superior à obtida bilateralmente, a comparação entre as médias mostrou-se limítrofe $(\mathrm{p}=0,053)$.

No que diz respeito aos dados de carga máxima, a tabela 3 exibe a comparação entre os braços esquerdo e direito. Tal como obtido na potência muscular máxima, não há diferença significativa $(p=0,20)$ e a correlação é praticamente perfeita $(r=0,99)$. Quanto à comparação entre o somatório de cargas máximas dos membros e as marcas obtidas com as cargas máximas desenvolvidas bilateralmente, verificou-se diferença significativa $(p=0,018)$. Na tabela 4 é demonstrada a comparação entre o somatório dos membros, com o trabalho bilateral na carga máxima. Em 54,2\% dos indivíduos o somatório das cargas máximas nos membros foi superior às cargas máximas obtidas nos trabalhos realizados bilateralmente.

\section{DISCUSSÃO}

A literatura referencia o fenômeno do déficit bilateral como leve diminuição da ativação neural no recrutamento de unidades motoras no desenvolvimento de trabalhos bilaterais, quando comparadas à soma dos trabalhos unilaterais ${ }^{19}$. Segundo Vandervoot et al. ${ }^{7}$, a capacidade de desen-

\section{TABELA 3}

Comparação da carga máxima entre os membros

\begin{tabular}{llllllll}
\hline \multicolumn{1}{c}{ Grupo } & N & Média & DP & Mediana & Mínimo & Máximo & EPM \\
Esquerdo & 24 & 29,3 & 13,9 & 30 & 11 & 54 & 2,8 \\
Direito & 24 & 29,7 & 14,2 & 29 & 12 & 55 & 2,8 \\
\hline
\end{tabular}

DP - desvio-padrão; EPM - erro padrão da média

TABELA 4

Comparação das cargas máximas no somatório dos membros com o trabalho bilateral

\begin{tabular}{lccccccc}
\hline \multicolumn{1}{c}{ Grupo } & N & Média & DP & Mediana & Mínimo & Máximo & EPM \\
& & & & & & & \\
Unilateral & 24 & 59,0 & 27,9 & 59 & 24 & 106 & 5,7 \\
Bilateral & 24 & 57,0 & 25,8 & 53 & 23 & 100 & 5,2 \\
\hline
\end{tabular}

DP - desvio-padrão; EPM - erro padrão da média 
volver força durante trabalhos isométricos e dinâmicos em baixas velocidades de forma bilateral, no exercício supino reto com halteres, apresentaram resultados similares quando comparados à somatória do trabalho unilateral. Outros estudos demonstram haver diferenças entre os membros inferiores e os superiores, assim como entre os próprios ${ }^{6,9,13,20}$, demonstrando assim a dificuldade em obtermos resultados similares nos estudos, provavelmente devido às diversas variáveis envolvidas. Uma das causas prováveis dessas diferenças seria a forma de avaliação através da eletromiografia, devido à sua baixa sensibilidade de avaliação em certos grupamentos em função do volume das massas musculares $^{21}$. Outros fatores como a capacidade de ativação neural, difusão dos impulsos entre os hemisférios cerebrais, estabilização postural, aprendizagem na coordenação, modulação aferente, redução da atividade do antagonista, motivação e tipo de fibra muscular envolvida ${ }^{22-26}$, devem ser levados em consideração.

Em nosso estudo foi encontrada uma diferença significativa no déficit bilateral em relação às cargas máximas, mas, em contraposição, o mesmo não ocorreu na potência muscular máxima. Já em um estudo proposto por Howard e Enoka ${ }^{21}$ nem todos os indivíduos apresentaram déficit bilateral, sendo que alguns demonstraram nenhuma diferença ou até mesmo facilitação bilateral. Em nosso estudo ocorreu o mesmo caso, sendo que $37,5 \%$ dos indivíduos apresentaram o trabalho bilateral na potência muscular máxima, superior ao somatório unilateral, e $21 \%$ dos avaliados apresentaram o trabalho bilateral, superior ao somatório unilateral na carga máxima.

A proposta deste estudo difere da maioria dos referenciados devido ao fato de apresentar dados referentes à comparação do déficit bilateral em uma avaliação muscular e não em um treinamento. A utilização do tensiômetro $F i$ trodyne também foi um diferencial, pois a maioria dos estudos foi realizada com aparelhos isocinéticos ${ }^{6,7,21,27,28}$. Uma possível limitação em nosso estudo, por não encontrarmos o déficit bilateral na potência muscular máxima, talvez tenha sido a metodologia realizada no teste de $1 \mathrm{RM}$. O incremento de cargas (dois quilos), talvez tenha sido grande, para o pequeno músculo testado, devido a esse grupamento apresentar alta sensibilidade em pequenos acréscimos de carga ${ }^{19}$. Outros estudos devem ser feitos com incrementos de cargas menores e com um número provavelmente maior de indivíduos, acarretando talvez resultados com diferenças significativas, devido aos nossos resultados terem ficado muito próximos à probabilidade estipulada para os critérios de significância.

Outro dado interessante foi em relação à carga alcançada na potência muscular máxima, em comparação com a carga máxima obtida em 1RM. Os valores do braço direito, braço esquerdo e ambos os braços, respectivamente, foram de 60 a $90 \%, 60$ a $89 \%$ e 65 a $96 \%$ de 1RM. Os escores obtidos demonstram que a carga na potência muscular máxima em pequenos grupamentos está próxima da carga máxima obtida com indivíduos destreinados, como também foi demonstrado em estudo anterior, no qual foi analisado o exercício remada em pé ${ }^{29}$. Contrariamente aos nossos achados, há dados na literatura sugerindo que cargas entre $30 \%$ a $60 \%$ de 1RM são as ideais para se trabalhar exercícios de potência ${ }^{8,12}$, contudo, em outros trabalhos, é demonstrado que em exercícios de potência muscular, essa relação pode chegar entre 70 a $88 \%$ de $1 \mathrm{RM}^{29,30}$. Contudo, diferenças entre os grupamentos musculares testados podem modificar os percentuais de carga que acarretem uma maior potência muscular ${ }^{8,12,29,30}$.

A comparação entre o braço esquerdo e o direito em cargas máximas e potência muscular máxima é limitada na literatura e dados do nosso estudo realizado em indivíduos jovens saudáveis demonstram não haver diferença significativa em nenhuma das duas variáveis avaliadas. Mesmo sendo todos os indivíduos destros, em 16,6\% dos indivíduos, a carga máxima direita foi menor que a carga máxima esquerda, e $37,5 \%$ desempenharam a mesma carga máxima para ambos os braços. Em relação à comparação de membros na potência muscular máxima, $41,6 \%$ dos indivíduos apresentaram uma potência muscular máxima direita inferior à da esquerda. Essas diferenças podem ser explicadas parcialmente, pela diferenças de fibras nos membros $^{31}$; recrutamento neural diferenciado pelo efeito cruza$\mathrm{do}^{32,33}$ ou pela predominância de utilização de um membro em detrimento de outro ${ }^{6,7}$. Conforme descrito por Gardiner ${ }^{19}$, o mecanismo pelo qual ocorre o déficit bilateral ainda permanece desconhecido.

Em conclusão, apesar de todos serem destros, não houve diferenças unilaterais em carga e potência muscular máximas, provavelmente devido à inexperiência nesse exercício. A soma dos resultados unilaterais difere em 5 a $10 \%$ daquele obtido bilateralmente, mostrando, contudo, tendências opostas entre potência muscular máxima e carga máxima, provavelmente refletindo uma limitação central na coordenação motora de um movimento complexo feito em máxima velocidade e com carga relativamente alta. Dependendo da situação-problema, se carga máxima ou potência muscular máxima, a estratégia de avaliação poderá ser diferente, assim como a interpretação dos resultados.

Em termos práticos de prescrição de exercícios de fortalecimento muscular pode-se optar igualmente por uma estratégia unilateral ou bilateral e especular que os resultados a serem obtidos com o treinamento serão semelhantes. 


\section{REFERÊNCIAS}

1. Friedrich MJ. Women, exercise, and aging. JAMA 2001;285:1429-31.

2. Foldvari M, Clark M, Laviolette LC, Bernstein MA, Kaliton D, Castaneda $\mathrm{C}$, et al. Association of muscle power with functional status in community-dwelling elderly women. J Gerontol A Biol Med Sci 2000; 55:192-9

3. Pollock ML, Gaesser GA, Butcher JD, Després JD, Dishman RK, Franklin BA, et al. The recommended quantity and quality of exercise for developing and maintaining cardiorespiratory and muscular fitness, and flexibility in healthy adults. Med Sci Sports Exerc 1998;30:975-91.

4. Evans WJ. Exercise training guidelines for the elderly. Med Sci Sports Exerc 1999;31:12-7.

5. Abernethy P, Wilson G, Logan P. Strength and power assessment. Issues, controversies and challenges. Sports Med 1995;19:401-17.

6. Vandervoot AA, Sale DG, Moroz J. Comparison of motor unit activation during unilateral and bilateral leg extension. J Applied Physiol 1984; 56:46-51.

7. Vandervoot AA, Sale DG, Moroz JR. Strength velocity relation and fatiguebility of unilateral versus bilateral arm extension. Eur J Appl Physiol 1987;56:201-5.

8. Fleck SJ, Kraemer WJ. Designing resistance training programs. $2^{\text {nd }}$ ed. Champaign: Human Kinetics, 1997:128-37.

9. Howard JD, Enoka RM. Maximum bilateral contractions are modified by neurally mediated interlimb effects. J Applied Physiol 1991;70:30616.

10. Patten C, Kamen G. Adaptations in motor unit discharge activity with force control training in young and older human adults. Eur J Appl Physiol 2000;83:128-43.

11. Secher NH. Isometric rowing strength of experienced and inexperienced oarsmen. Med Sci Sports 1975;7:280-3.

12. Kraemer WJ, Fleck SJ, Evans WJ. Strength and power training; physiological mechanisms of adaptation. Exer Sport Sci Rev 1996;24:363-97.

13. Kroll W. Central facilitation in bilateral versus unilateral isometric contractions. Am J Phys Med 1965;44:218-23.

14. Feigenbaum MS, Pollock ML. Strength training-rationale for current guidelines for adult fitness program. Phys Sports Med 1997;25:44-64.

15. Shepard RJ. PAR-Q, Canadian home fitness test and exercise screening alternatives. Sports Med 1988;5:185-95.

16. De Lorme TL, Watkins AL. Technics of progressive resistance exercise. Arch Phys Med 1948;29:263-73

17. Monteiro WD. Medidas da força muscular, aspectos metodológicos e aplicações. Treinamento Desportivo 1998;1:38-51.

18. Gordon CC, Chunlea WC, Roche AF. Stature, recumbent length, and weight. In: Lohman TG, Roche AF, Martorell R, editors. Antropometric standardization reference manual. Champaign: Human Kinetics, 1988: $3-8$.

19. Gardiner PF. Neuromuscular aspects of physical activity. Champaign: Human Kinetics, 2001:78.

20. Schantz PG, Moritani T, Karlson E, Johansson E, Lundh A. Maximal voluntary force of bilateral and unilateral leg extension. Acta Physiol Scand 1989;136:185-92.

21. Howard JD, Enoka RM. Maximum bilateral contractions are modified by neurally mediated interlim effects. J Appl Physiol 1991;70:306-16.

22. Lynch NA, Metter EJ, Lindle JL, Fozard JL, Tobin JD, Roy TA, et al. Muscle quality. I age associated differences between arm and leg muscle groups. J Apllied Phys 1999;86:188-94

23. De Ruiter CJ, Jones DA, Sargeant AJ, De Haan. The measurement of force/velocity relationships of fresh and fatigued human adductor pollicis muscle. Eur J Appl Physiol 1999;80:386-93.

24. Kanehisa H, Miyashita M. Specificity of velocity in strength training. Eur J Appl Physiol 1983;52:104-6.

25. Rutherford OM, Jones DA. The role of learning and coordination in strength training. Eur J Appl Physiol 1986;55:100-5.

26. Zhou S. Chronic neural adaptations to unilateral exercise: mechanisms of cross education. Exerc Sport Sci Rev 2000;28:177-84.

27. Coyle EF, Feiring DC, Rotkis TC, Cote RW, Roby FB, Lee W, et al. Specificity of power improvements through slow and fast isokinetic training. J Appl Physiol 1981;51:1437-42.

28. Jakobi JM, Cafarelli E. Neuromuscular drive and force production are not altered during bilateral contractions. J Appl Physiol 1998;84:200-6.

29. Simão R, Monteiro WD, Araújo CGS. Fidedignidade inter e intradias de um teste de potência muscular. Rev Bras Med Esporte 2001;7:118-24.

30. Hoeger WWK, Hopkins DR, Barette SL, et al. Relationship between repetitions and selected percentages of one repetition maximum: a comparison between untrained and trained males and females. J Appl Sports Sci Res 1990;4:47-54.

31. Moritani T, De Vries HA. Neural factors versus hypertrophy in the time course of muscle strength gain. Am J Phys Med 1979;58:115-31.

32. Hakkinen K, Kallinen M, Linnamo V, Pastinen UM, Newton RU, Kraemer WJ. Neuromuscular adaptations during bilateral versus unilateral strength training in middle-aged and elderly men and women. Acta Physiol Scand 1996;158:77-88.

33. Hortobágyi T, Lambert NJ, Hill JP. Greater cross education following training with muscle lengthening than shortening. Med Sci Sports Exerc 1997;29:107-12. 